\title{
Percepción de profesores-tutores y maestrantes de la DAEA-UJAT, en cuanto a las competencias y funciones que deben desarrollar éstos en el proceso formativo de los estudios de posgrado
}

\author{
Perception of teachers and masters students from DAEA-UJAT, regarding \\ the competences and functions which they must develop during the \\ formative process in a postgraduate course of study
}

Percepção de professores-tutores e docentes do DAEA-UJAT, quanto às competências e funções que devem desenvolver no processo de formação de pós-graduados

Adriana Esperanza Pérez Landero Universidad Juárez Autónoma de Tabasco, México adriana.landero@yahoo.com.mx https://orcid.org/0000-0001-9058-3411

\section{Resumen}

El objetivo de este trabajo fue conocer la opinión de profesores, estudiantes y coordinadores de dos maestrías relacionadas con la educación en torno a las funciones que debe cumplir un tutor, rol esencial para que los maestrantes puedan culminar con éxito el proceso formativo que siguen en un posgrado. Para ello, la presente investigación se sustentó en una visión holística e integral de la acción tutorial y en el método de la teoría de roles (Deutsch y Krauss, 2001). Los participantes fueron estudiantes, docentes y coordinadores de dos maestrías de la División Académica de Educación (DAEA) y Artes de la Universidad Juárez Autónoma de Tabasco. Con base en las opiniones recabadas, se puede inferir que todos los implicados en los programas de posgrado seleccionados expresaron que es indispensable que la DAEA cuente con un programa de capacitación para tutores de posgrado que se enfoque en el estudio, la consolidación y el desarrollo de las funciones, roles, perfiles, organización, currículos y aspectos psicopedagógicos que se vinculan con la acción tutorial. 
Palabras claves: Estrategia, formación, pedagógica, posgrado, tutores, tutorías.

\begin{abstract}
The objective of this work was to know the opinion of teachers, students and coordinators from two different masters related to education regarding to the functions that a tutor must fulfill; essential role so the teachers can successfully conclude the formative process in a postgraduate course of study. In order to do that, the present investigation was based on a holistic and integral visión from the tutorial action and the method of role theory (Deutsch and Krauss, 2001). The participants were teachers, students and coordinators from two different masters of la División Académica de Educación (DAEA) y Artes from Universidad Juárez Autónoma de Tabasco.

Based on the opinions collected, it can be inferred that everyone involved and selected in the programs of this a postgraduate course pointed out that it is indispensable for DAEA to have a training program for graduate mentors focused on the study, consolidation and development of the functions, roles, profiles, organization, curricula and psychopedagogical aspects that are linked to the tutorial action.
\end{abstract}

Keywords: strategy, training, pedagogical, postgraduates, tutors, tutorials.

\title{
Resumo
}

O objetivo deste estudo foi conhecer a opinião de professores, alunos e coordenadores de dois diplomas de mestrado relacionados com a educação em torno das funções a serem cumpridas por um papel essencial tutor para o Grandee pode concluir com êxito o processo de formação após uma pós-graduação Para o efeito, esta pesquisa foi baseada em uma visão holística e abrangente do tutorial e o método da teoria do papel (Deutsch e Krauss, 2001). Os participantes eram estudantes, professores e coordenadores de graus Divisão de dois de mestrado acadêmico Educação (DAEA) e Artes da Universidad Autonoma de Juarez Tabasco. Com base nas opiniões recolhidas, podemos inferir que todos os envolvidos nos programas de pós-graduação selecionados afirmou que é essencial que a contagem DAEA em um programa de formação de 

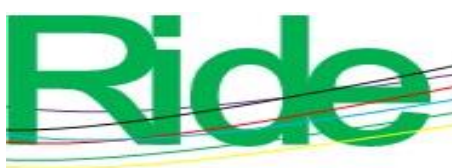

Revista Iberoamericana para la Investigación y el Desarrollo Educativo ISSN 2007 - 7467

professores de pós-graduação se concentra no estudo, consolidação e desenvolvimento de funções, papéis, perfis, organização curricular e aspectos de psicologia educacionais relacionados com o tutorial.

Palavras-chave: Estratégia, formação, pedagogia, pós-graduação, tutores, tutoriais.

\section{Introducción}

La globalización y el reordenamiento paradigmático de la actualidad exigen transformaciones sustanciales en el ámbito educativo para que las personas se puedan desenvolver con éxito en el mercado laboral. En este sentido, instituciones como la Organización para la Cooperación y Desarrollo Económico (OCDE) (2008) han realizado en México esfuerzos para que se concreten adaptaciones y reformas necesarias a los programas educativos del país, especialmente de posgrado, pues la responsabilidad de este nivel formativo es brindar a la sociedad las herramientas indispensables para transformar la realidad. Esto se logra desarrollando en los profesionales sus capacidades de docencia, extensión, investigación y, fundamentalmente, tutoría, rol esencial para el asesoramiento disciplinar, indagatorio y científico de los especialistas.

Para esto, no solo se debe contar con los recursos financieros, la infraestructura, la tecnología y el acervo bibliográfico en un marco de políticas y decisiones que incidan en la dinámica de organizar, transmitir y producir el saber, sino que también se debe impulsar una cultura basada en la investigación, donde los tutores con una mayor producción académica e investigativa puedan compartir sus conocimientos. En el caso concreto de México, de hecho, se han reconocido algunos de los elementos clave que se pueden impulsar para trabajar en la formación de los tutores, por ejemplo:

- Fines de formación.

- Concepción de aprendizaje.

- Procesos metodológicos.

- Desarrollo de competencias. 
- Alternativas de acción y comunicación.

- Condiciones académico-administrativas.

Estos criterios de formación, sin embargo, contrastan con algunos programas que se han creado producto de una serie de talleres vivenciales que han intentado descubrir cuáles de los elementos antes mencionados han impactado en sus posgrados.

\section{Antecedentes}

En todas estas Instituciones de Educación Superior, por estatuto se estableció la función Tutorial como una actividad más de los profesores, con la finalidad de apoyar de manera integral a los alumnos y a su vez lograr una calidad y eficiencia educativa, todo ello en respuesta a la perspectiva que planteaban organismos internacionales enfocados a dicho sector, como la UNESCO en su declaración Mundial sobre la Educación Superior en el Siglo XXI: Visión y Acción (1998) quien considera que el personal docente debe estar disponible para los alumnos, con el propósito de orientarles en sus estudios. Se señalándose entre las diversas responsabilidades que debe asumir el maestro, el de "proporcionar cuando proceda, orientación y consejo, cursos de recuperación, formación para el estudio y otras formas de apoyo a los estudiantes, comprendidas las medidas para mejorar sus condiciones de vida" (ANUIES, 2000)

Obviando los intentos improvisados por trabajar en la formación de tutores, a continuación, se describen una serie de estudios y experiencias sistematizadas que de forma genuina se han preocupado por formar de la mejor manera posible a los tutores. En tal sentido, se puede nombrar el caso de la Universidad Nacional Autónoma de México (UNAM), donde se ha ofrecido un taller para tutores del posgrado en Ciencias de la Administración, el cual fue aplicado a un grupo de doce tutores de maestría y doctorado durante un periodo de 15 horas. La finalidad de este fue recabar experiencias e intercambiar opiniones para sistematizar y especificar las funciones, los conocimientos, las habilidades, las actitudes y los requerimientos necesarios para desempeñarse con éxito en dicho rol; contribuyendo al reconociendo de la importancia del proceso de las prácticas de tutoría, como contribución al desarrollo y consolidación del posgrado en ciencias experimentales a través del análisis de las prácticas y los procesos de formación. 

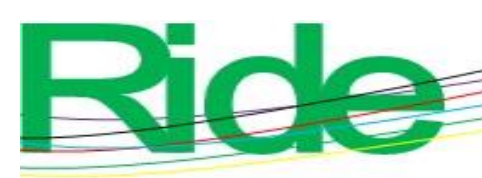

Revista lberoamericana para la

Investigación y el Desarrollo Educativo ISSN $2007-7467$

Los resultados de este trabajo demostraron que uno de los factores más decisivos para el desarrollo del posgrado era la tutoría, ya que esta potencia la autonomía de los estudiantes a través de un modelo de enseñanza que procura ser directo, inmediato y personal.

Igualmente, sobresale el trabajo Competencias académicas de los tutores del posgrado en ciencias de la tierra de la UNAM, GEOS, Unión Geofísica Mexicana, A.C., realizado por García, Laguna, Campos, Ruiz y Martínez (2001), en la maestría y doctorado en Ciencias de la Tierra. Esta indagación consistió en analizar cada una de las funciones, actividades, conocimientos, habilidades, actitudes y valores requeridos para desempeñar con éxito la actividad tutorial, de las cuales sobresalieron las competencias vinculadas con la investigación, la comunicación, la previsión y el trato personal, así como el desempeño de sus funciones en el proceso de orientación del tesista.

Otro estudio importante lo constituye el realizado en la UNAM por De la Cruz y Abreu (2017), quienes evaluaron la complejidad de los procesos de tutoría en el nivel de posgrado, lo que los llevó a plantear y clarificar los roles y las funciones que ellos deben de asumir en su labor tutorial.

Por otra parte, en La asesoría de tesis en el posgrado, López y Farfán (2005) dieron a conocer que el proceso de asesoría de tesis y trabajos de grado estaba influenciado por el contexto social, la institución y la falta de motivación de los participantes, mientras que Evaluación de la tutoría en los estudios de posgrado: construcción y validez (De la Cruz y Abreu 2017) identificaron algunos de los requerimientos que exigían los alumnos de sus futuros tutores. Este trabajo se realizó mediante entrevistas a una muestra de 194 aspirantes a ingresar a los programas de Ciencias Médicas y Ciencias de la Salud de la UNAM. Los resultados evidenciaron que los participantes esperaban de sus tutores habilidades en el campo de la docencia, así como sensibilidad para fungir como consejeros académicos en el trascurso de la investigación.

Expectativas de profesores y estudiantes de posgrado frente a la tutoría (López \& Ortega 2006) de la Universidad de Guadalajara, se centró en la riqueza de las expectativas de cada uno de estos actores en lo que esperan y contempla sea la tutoría en este nivel; tomando los aspectos de: tiempo, orientación, publicación, investigación, Tesis, entre otros. 


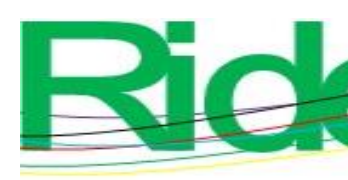

Revista lberoamericana para la

Investigación y el Desarrollo Educativo ISSN $2007-7467$

Ahora bien, en el caso específico de la Universidad Juárez Autónoma de Tabasco (UJAT) vale destacar que si bien en esta casa de estudios se contempla el sistema tutorial en los diversos programas de posgrado de la División Académica de Educación y Artes (DAEA) y en el Plan Estratégico de Desarrollo 2006-2016, en el Plan de Desarrollo Institucional 2012-2016, así como en los reglamentos de estudios y lineamientos curriculares, la realidad indica que en la práctica no existe un claro seguimiento de esta importante labor.

Debido a esto, se pretende conocer si los docentes de los programas de la DAEA cuentan con la formación necesaria para ejercer el rol de tutores. Planteándose las interrogantes: ¿cuáles son las tendencias internacionales y nacionales en relación con la función de las tutorías? y ¿cuáles son los fundamentos teóricos que sustentan la formación y funciones que debe asumir el tutor que se desempeña en los programas de maestría de la DAEA-UJAT para contribuir a un proceso formativo de calidad en los estudiantes que cursan sus maestrías?

\section{Roles del tutor}

Uno de los roles más significativos para cualquier posgrado lo desempeñan los tutores, pues sobre estos profesionales recaen muchas de las responsabilidades que se relacionan con la orientación, el seguimiento y la culminación exitosa de la investigación que deben desarrollar todos los estudiantes. Para esto, el tutor debe ser un profesional integral, ya que debe estar capacitado no solo en el área de investigación escogida por el estudiante, sino también en otras facetas relacionadas con los aspectos psicosociales y las relaciones interpersonales. Esto se evidencia en los distintos roles que un tutor debe cumplir, los cuales se enumeran a continuación:

1. Rol de docente: Facilita la adquisición, construcción y sistematización del conocimiento, así como de habilidades intelectuales y procedimentales.

2. Rol de apoyo psicosocial: Ofrece ayuda psicológica para resolver, junto con el tutorado, situaciones conflictivas durante su formación. Igualmente, alienta su desarrollo personal y profesional.

3. Rol de entrenador (coach): Entrena en la adquisición de habilidades intelectuales, pragmáticas y de investigación. 
4. Rol de consejero académico: Actúa como enlace entre el tutorado y el programa de estudio en cuestiones de normas, planificación, financiamientos, becas, requisitos de egreso, etc.

5. Rol de patrocinador: Ayuda a través de su conocimiento en el área, prestigio y red de colegas para que el tutorado tenga mejores oportunidades para desarrollar su investigación, presentar sus hallazgos en comunidades académicas y obtener mejores promociones laborales.

6. Rol de socializador: Transfiere las normas, valores, prácticas, actitudes, etc., de la profesión e incorpora al tutorado a grupos de pares y expertos.

7. Rol de investigador: Estimula la formación de actitudes intelectuales y morales hacia la ciencia y contribuye a la búsqueda de la verdad científica.

Estos roles, por supuesto, se pueden desarrollar a través de diversas actividades (p. ej., cursos, talleres, diplomados, etc.) que pueden brindar al tutor una serie de elementos teóricos, prácticos y metodológicos que le permitirán cumplir con una acción tutorial integral, es decir, sustentada no solo en el conocimiento de herramientas tecnológicas y metodológicas propias de determina disciplina, sino también en el desarrollo de principios y valores humanos, los cuales también son indispensables para facilitar el proceso de orientación investigativa.

Si bien es cierto que estos cambios deben de estar presentes en todos los niveles educativos, mención especial requiere el nivel superior y estudios de posgrado los cuales hoy enfrentan grandes retos, en el proceso formativo de sus educandos, ya que a estas instituciones educativas la sociedad les ha asignado la misión de ser los espacios en donde se formen los cuadros humanos de profesionistas que generen y difundan conocimientos basados en un desarrollo integral, Específicamente los estudios de postgrado destacan en este compromiso de las IES, por lo que "han adoptado la investigación científica como su objetivo esencial, considerando que esto refleja realmente una visión estrecha de la ciencia y las potencialidades y necesidades de desarrollo intelectual del hombre y de la sociedad..."(Balmaseda \& Castro 2010). 

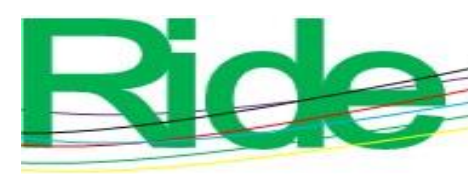

Revista Iberoamericana para la

Investigación y el Desarrollo Educativo ISSN $2007-7467$

Esto se encuentra en concordancia con lo planteado en el Programa Nacional de Posgrados de Calidad (PNPC) de México, donde se destaca la consolidación del posgrado a través de la formación de sus cuadros de investigadores y profesionistas para brindar una educación de calidad (Consejo Mexicano de Estudios de Posgrado [Comepo], 2005). En este sentido, se debe recalcar que la formación al más alto nivel de los tutores también incide en la instrucción de los futuros posgraduados.

La acción tutorial, por tanto, debe enfocarse en generar alternativas de atención que incidan en una formación integral de los tutorados, lo cual se puede conseguir a través de la producción científica, la creación de redes de investigación y académicas, así como mediante la dirección, el desarrollo y la obtención del grado. En este proceso, los tutores deben crear espacios en los cuales los estudiantes puedan, de una manera sistemática e intencionada, asimilar, ensayar y experimentar con determinadas tareas, las cuales deben enfocarse en conseguir un profesional consciente de su propia práctica para dar respuesta a los problemas de su realidad.

En México el Consejo Nacional de Ciencia y Tecnología menciona sobre el eje enfocado a la Tutoría, que es un rubro considerado como un elemento de calidad y requisito evaluador indispensable con el que deben de contar todos los estudios de posgrado, para ser evaluados por los organismos evaluadores y certificadores, ya que la Tutoría desempeña un papel primordial que permitirá el desarrollo y conclusión en tiempo y forma de los estudios en este nivel, por ser una acción que facilita, guía y forma a los alumnos durante su formación académica (CONACyT, 2010)

Planteado lo anterior, se puede afirmar que la presente investigación se sustenta en una visión holística e integral de la acción tutorial y en el método de la teoría de roles donde tanto el tutor como el estudiante tienen diferentes funciones que deben desarrollar en un proceso complejo y dinámico (Deutsch y Krauss, 2001, De la Cruz y Abreu, 2004). 


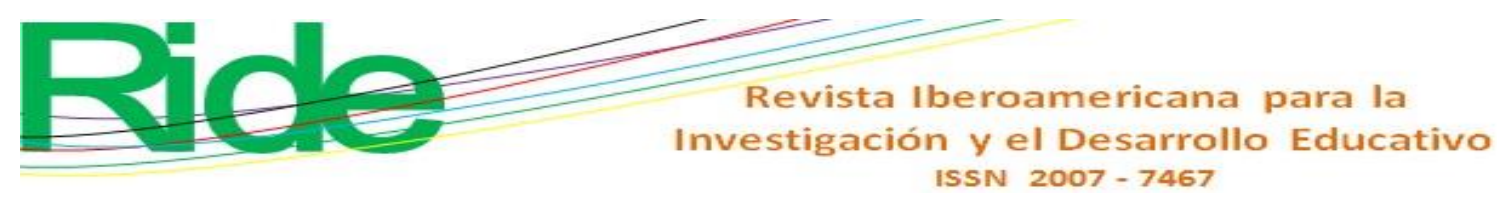

\section{Materiales y métodos}

Esta investigación se sustentó en el paradigma cuantitativo y cualitativo, (integral, bimodal), puesto que se abordó el objeto de estudio desde un modelo mixto, el cual representa el más alto grado de integración de ambos enfoques. Los participantes elegidos fueron los siguientes:

- 23 estudiantes de $2 .^{\circ}$ y $4 .^{\circ}$, sección A, de la maestría en Docencia de la DAEA de la UJAT.

- 19 estudiantes de $1 .^{\circ}$ y $3 .^{\circ}$, sección A, de la maestría en Educación de la DAEA de la UJAT.

- 7 docentes y 2 coordinadores de las mencionadas maestrías.

Los métodos teóricos utilizados fueron la modelación científica, que facilitó obtener el modelo de la estrategia pedagógica y las relaciones existentes entre sus diversos componentes. Asimismo, del método empírico se utilizó el preexperimento con el fin de valorar el grado de conocimiento y compromiso de los tutores por ejercer su labor según el contexto estudiado.

Para ello, se emplearon instrumentos como el cuestionario, el cual "consiste en un conjunto de preguntas respecto de una o más variables a medir" (Hernández, Fernández y Baptista, 2014, pág. 217) Este se usó para conocer la opinión y las expectativas de los estudiantes en relación con la responsabilidad, los conocimientos, el perfil de aptitudes y las funciones que esperan de sus tutores. Esto quiere decir que el cuestionario quedó estructurado con las siguientes cuatro unidades de análisis:

a. Conceptualización de la acción tutorial en posgrado.

b. Perfil, rol y función del tutor.

c. Experiencia personal de su proceso tutorial.

d. Ventajas y desventajas de ser tutorado.

El cuestionario final quedó conformado por un total de 18 interrogantes distribuidas en preguntas de opción múltiple, dicotómicas, de cascadas, cerradas y abiertas. 

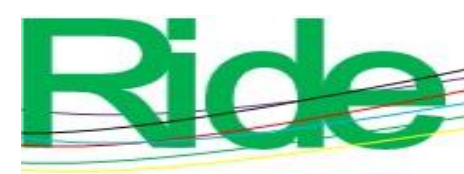

Revista lberoamericana para la

Investigación y el Desarrollo Educativo ISSN $2007-7467$

El segundo instrumento empleado fue la entrevista cualitativa cara a cara, puesto que brinda la oportunidad de conocer a profundidad la opinión de los sujetos entrevistados. En concreto se trabajó con la entrevista semiestructurada, la cual se basa "en una guía de asuntos o preguntas y el entrevistador tiene la libertad de introducir preguntas adicionales para precisar conceptos u obtener mayor información sobre los datos deseados" (Hernández, Fernández y Baptista, 2014, pag. 403) Este instrumento se usó para conocer cuál era la percepción que tenían los profesores sobre la acción tutorial en el marco de los programas formativos de la DAEA-UJAT. Esta entrevista, por tanto, quedó diseñada con un total de 20 preguntas que se enfocaron en los siguientes aspectos:

a. Datos de identificación profesional.

b. Conceptualización de la acción tutorial en posgrado.

c. Perfil, rol y función de un tutor.

d. Experiencia personal de su proceso tutorial.

e. Diferencias entre tutor y asesor.

f. Ventajas y desventajas de la acción tutorial en posgrado.

Con ambos instrumentos se realizó una prueba piloto para validar su confiabilidad y para realizar las correcciones necesarias. Luego, con los datos recabados en los instrumentos definitivos, se procedió a modelar una estrategia de formación de tutores de posgrado, la cual se sometió a la evaluación de expertos. La composición del grupo de especialistas fue de 10 docentes (6 maestros y 4 doctores en las disciplinas de Educación, Gestión, Planeación y Administración Educativa, Psicología y Estudios Organizacionales). Todos ellos tenían más de 6 años de experiencia como docentes en el nivel de posgrado. 


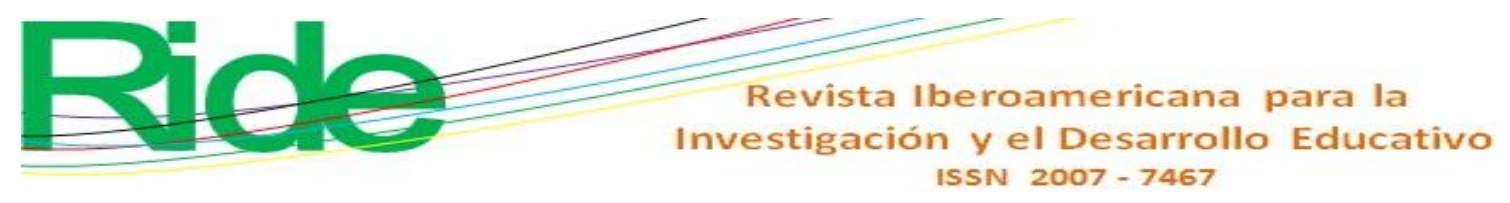

\section{Resultados}

A partir de los resultados comparativos de los cuestionarios aplicados a los maestrantes, se puede afirmar que $45 \%$ de estudiantes de $1 .{ }^{\text {er }}$ semestre y $67 \%$ de 3 . $^{\text {er }}$ semestre de la maestría en Educación, así como $50 \%$ de $2 .^{\circ}$ semestre y $78 \%$ de $4 .^{\circ}$ semestre de la maestría en Docencia consideraron que la tutoría es una labor que debe servir como apoyo integral de largo alcance, cuyos propósitos y metas deben incidir en una mejor formación y calidad educativa en los posgrados.

Por otra parte, en cuanto al perfil del tutor, $50 \%$ de estudiantes de $1 .^{\mathrm{er}}$ semestre y $37 \%$ de $3 .^{\text {er }}$ semestre de la maestría en Educación, así como $67 \%$ de $2 .^{\circ}$ semestre y 67 $\%$ de $4 .^{\circ}$ semestre de la maestría en Docencia opinaron que el tutor debía poseer experiencia docente y de investigación, así como un equilibrio entre la relación afectiva y cognoscitiva para tener capacidad y dominio del proceso de la tutoría.

Aunado a ello, los profesores que laboran en el posgrado señalaron que los tutores deben tener experiencia en docencia e investigación, y contar con el grado equivalente en el cual se encuentran colaborando. Asimismo, formar parte de las redes de colaboración para vincular a los maestrantes en la investigación, y estar interesados en responsabilizarse por un número determinado de alumnos. Igualmente, tener ética profesional, estar en constante actualización y trabajar de forma interdisciplinaria para crear, transformar y difundir el conocimiento en la sociedad.

Por otra parte, todos los encuestados de $1 .^{\text {er }}$ y 3 er $^{\text {er }}$ semestre de la maestría de Educación, así como todos los de $2{ }^{\circ}$ semestre de la maestría en Docencia expresaron que sí les interesaría contar con un tutor durante la formación de posgrado, mientras que $11 \%$ de los estudiantes de $4 .^{\circ}$ semestre de Docencia no estaban seguros de contar con este apoyo.

Sobre los beneficios de estas tutorías, $37 \%$ de estudiantes de $1 .{ }^{\text {er }}$ semestre y 60 $\%$ de $3 .^{\text {er }}$ semestre de la maestría en Educación, así como $56 \%$ de $2 .^{\circ}$ semestre y $22 \%$ de $4 .^{\circ}$ semestre de la maestría en Docencia manifestaron que serviría para incentivar sus capacidad para redescubrir nuevas alternativas ante las inquietudes-conocimientos, lo cual los potenciaría en las diversas áreas disciplinarias en que se forman.

En lo que respecta a la tutoría y asesoría, $80 \%$ de estudiantes de 1 . $^{\text {er }}$ semestre y $100 \%$ de $3 .^{\text {er }}$ semestre de la maestría en Educación, así como $90 \%$ de $2 .^{\circ}$ semestre y 67 $\%$ de $4 .^{\circ}$ semestre de la maestría en Docencia consideró que existían diferencias entre 

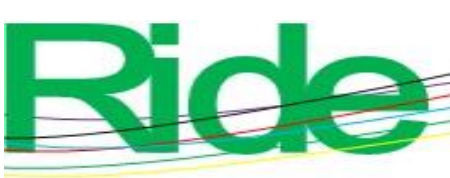

Revista lberoamericana para la

Investigación y el Desarrollo Educativo ISSN $2007-7467$

ambas acciones, pues la tutoría implica un proceso más complejo, integral y humanista en cuanto a la información del sujeto, mientras que la asesoría solo se enfoca en guiar en algunos aspectos de la tesis.

Por su parte, los profesores entrevistados mencionaron que la figura del tutor no se puede fusionar con la del asesor, puesto que ambas, aunque complementarias, deben aplicarse por separado; en este sentido, opinaron que el asesor cumpliría la labor de investigación con el alumno, mientras que el tutor abarcaría en un sentido más amplio dicha labor, sin especificar cuál sería el rol o la labor del tutor en ese proceso. Para el experto la figura del tutor y el asesor no se deben confundir, pues el tutor desempeña su papel de manera más personal con la intención de trabajar en la formación de los alumnos y en la consecución de la titulación.

Igualmente, cabe resaltar que los profesores entrevistados comentaron que la falta de tiempo impide cumplir con las tareas de tutorías, de modo que la mayoría de ellos solo fungen como guías en el desarrollo de las tesis, aunque con el paso del tiempo dejan de hacerlo. Igualmente, señalaron que esta función no aparece establecida en los programas institucionales de los posgrados, por lo que se da libertad para ejercerla o dejarla al margen. Por este motivo, se deben crear escenarios que permitan contar con tutores, pues de esta manera se elevaría la eficiencia terminal y de titulación del posgrado, así como la calidad formativa de los maestrantes.

En cuanto al tipo de atención de los tutores, $50 \%$ de estudiantes de $1 .{ }^{\mathrm{er}}$ semestre y $50 \%$ de $3 .^{\text {er }}$ semestre de la maestría en Educación consideraron que esa debería ser una acción individualizada, mientras que $67 \%$ de estudiantes de $4 .^{\circ}$ semestre de la maestría en Docencia expresaron que requerían a un tutor para la guía en los proyectos de investigación. Esta queja se justifica porque los maestrantes deben entregar un proyecto de investigación como parte final de su formación de posgrado.

Por este motivo, se les consultó a los estudiantes si les gustaría que hubiese un programa de tutorías para los estudios de posgrado. Los datos recabados indicaron que todos los encuestados apoyarían ese tipo de iniciativas, aunque vale comentar que $11 \%$ de los maestrantes del 4. ${ }^{\circ}$ semestre en Docencia respondieron de forma negativa.

Sobre este cuestionamiento, se debe agregar la opinión de los profesores, quienes indicaron que no habían recibido capacitación específica de tutorías para nivel de posgrado, aunque sí para el nivel de licenciatura. Asimismo, agregaron que su trabajo como docentes en las mencionadas maestrías fue requerido por la institución. 

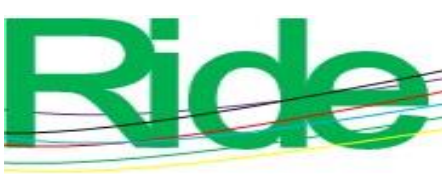

Revista Iberoamericana para la

Investigación y el Desarrollo Educativo ISSN $2007-7467$

En cuanto a los directivos entrevistados, se puede decir que estos opinaron que las funciones que debían cumplir los tutores se relacionaban con la orientación bibliográfica y la guía de trabajos de investigación. Asimismo, consideraron que los tutores deberían ser profesores de tiempo completo que cuenten con proyectos de investigación registrados o institucionalizados; incluso pertenecer a redes de colaboración de vinculación con otros académicos nacionales, así como tener habilidad para identificar y ofrecer una orientación en relación con los aspectos académicos, emocionales y psicológicos del estudiante.

\section{Conclusiones}

Con base en las opiniones recabadas, se puede inferir que todos los implicados en las maestrías seleccionadas en este trabajo expresaron que es indispensable que la DAEA cuente con un programa de capacitación para tutores de posgrado que se enfoque en el estudio, la consolidación y el desarrollo de las funciones, roles, perfiles, organización, currículos y aspectos psicopedagógicos que se vinculan con la acción tutorial.

Una propuesta de formación de tutores, por tanto, debe partir del diagnóstico situacional y del análisis de los lineamientos curriculares de las maestrías analizadas. De esta manera, se puede trabajar en la sensibilización sobre la importancia de la acción tutorial y proponer ejes de acción que se enfoquen en el ámbito investigativo y disciplinar. Asimismo, estipular de manera obligatoria y bien definida dentro de los currículos institucionales de estos programas las responsabilidades que debe desempeñar el tutor, pues de esta forma los maestrantes y tutores podrán cumplir con el nivel de compromiso y de calidad establecidos. Esto permitirá fortalecer la preparación de la planta docente de los posgrados en materia de la acción tutorial.

En efecto, los programas de posgrado, en general, exigen de los profesores amplias competencias no solo en el campo de la docencia, sino también en la investigación y en las relaciones humanas. Para esto, se deben fomentar programas que procuren conseguir la pertinencia, la equidad y la calidad para contribuir al desarrollo de procesos de gestión que impacten en la mejora continua. En este sentido, se debe dar importancia a los contenidos de aprendizajes y a la forma de desarrollarlos, con 

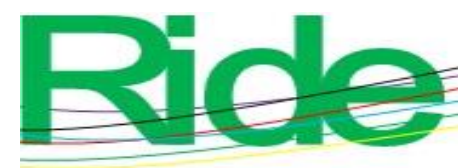

Revista Iberoamericana para la

Investigación y el Desarrollo Educativo ISSN 2007 - 7467

docentes-tutores que conduzcan de manera eficiente y eficaz, y con bases teóricas y prácticas el proceso de enseñanza y aprendizaje.

El docente-tutor, por tanto, debe saber emplear los elementos teóricos, psicopedagógicos, didácticos e instrumentales para crear condiciones reales que permitan al estudiante de posgrado potenciar sus conocimientos y desarrollar su creatividad, responsabilidad, compromiso, sensibilidad y conciencia.

Esto quiere decir que la tutoría en posgrado debe ser entendida como una actividad de carácter formativo y dinamizador del aprendizaje de los alumnos, basada en un modelo flexible e integrador, que permita interrelacionar funciones para impulsar una formación integral basada en la proyección social y profesional de los egresados.

Por eso, en el caso concreto de la DAEA, es urgente que los profesores de posgrado conciban de forma positiva el proceso de la acción tutorial, pues de esa manera se pueden romper las barreras que frenan los procesos de formación académica de los alumnos. Esto significa que el docente de posgrado de la DAEA debe reconocer que la acción tutorial es una exigencia continua de la formación profesional en la cual convergen implicaciones de índole académica, personal y vocacional. 


\section{Referencias}

Asociación Nacional de Universidades e Instituciones de Educación Superior (ANUIES) (2000). Programas institucionales de tutoría: una propuesta de la ANUIES para su organización y funcionamiento en las instituciones de educación superior. México: Series Investigaciones. http://evirtual.uaslp.mx/FCQ/tutorias/Documentos\%20compartidos/INTRODUC CION/PROGRAMAS\%20INSTITUCIONALES\%20DE\%20TUTORIA_ANUI ES.pdf

Balmaseda, N., Polaino de los Santos, O. y Castro Lamas, J. (2010). Hacia una gestión eficiente del postgrado. Tendencias, motivación y satisfacción de necesidades. Trabajo presentado en el Congreso Universidad 2010. Curso Precongreso n. ${ }^{\circ} 5$. Cuba. (pp. http://beduniv.mes.edu.cu/fetch.php?data=352\&type $=$ pdf $\& \mathrm{id}=2965 \& \mathrm{db}=0$

Consejo Nacional de Ciencia y Tecnología (Conacyt) (2015). Programa Nacional de Posgrados de Calidad. Marco de referencia para la evaluación y seguimiento de programas de posgrado. México. https://www.uv.mx/posgrado/files/2012/11/MARCO-DE-REFERENCIAPNPC-V6.pdf

Consejo Mexicano de Estudios de Posgrado [Comepo] (2005). Plan de desarrollo del postgrado nacional.

De la Cruz, F. G. y Abreu, H. L. (2017). Evaluación de la tutoría en los estudios de posgrado: construcción y validez de escalas. (pp. 1-26) Vol.15. Universidad Nacional Autónoma de México. https://dialnet.unirioja.es/descarga/articulo/6275386.pdf

García, M., Laguna, J., Campos, J., Ruiz, R., \& Martínez, A. (2001). Competencias académicas de los tutores del posgrado en ciencias de la tierra de LA UNAM, GEOS, Unión Geofísica Mexicana, A.C., Abril. https://www.ugm.org.mx/publicaciones/geos/pdf/geos01-1/Garcia-Sahagun01-1.pdf

Hernández, R., Fernández, C. y Baptista, P. (2014). Metodología de la investigación (6. ${ }^{a}$ ed.). McGraw-Hill, México. 


\section{Revista Iberoamericana para la Investigación y el Desarrollo Educativo ISSN 2007 - 7467}

López, O. A. (2006). La tutoría en los posgrados: una delimitación conceptual andragógica. México: Centro Universitario de Ciencias de la Salud, Universidad de Guadalajara.

López, O. y Farfán, F. (2005). La asesoría de tesis en el posgrado. Trabajo presentado en el VIII Congreso Nacional de Investigación Educativa, Hermosillo Sonora.

Organización para la Cooperación y el Desarrollo Económicos (OCDE). (2008). Panorama de la $\quad$ Educación $2008 . \quad$ Recuperado de:http://www.oecd.org/centrodemexico/medios/41288187.pdf 